\title{
Effect of Hatha yoga training on rhinitis symptoms and cytokines in allergic rhinitis patients
}

\author{
Anan Chanta, ${ }^{1}$ Jettanong Klaewsongkram, ${ }^{2}$ Timothy D. Mickleborough, ${ }^{3}$ Wannaporn Tongtako ${ }^{1}$
}

\begin{abstract}
Introduction: Allergic rhinitis is an inflammation of the nasal mucosa in response to allergens. There is evidence that yoga can improve personal health and has positive effects on immune function. However, the effects of Hatha yoga training on rhinitis symptoms and cytokines in patients with allergic rhinitis are still unclear.
\end{abstract}

Objective: The purpose of this study was to investigate the effects of Hatha yoga training on rhinitis symptoms and cytokines in allergic rhinitis patients.

Methods: Twenty-seven allergic rhinitis patients were randomized into 2 groups: a control group $(\mathrm{CON} ; \mathrm{n}=14)$ and a yoga group (YOG; $\mathrm{n}=13$ ). The CON group continued with normal activities and the YOG group was required to complete a protocol of Hatha yoga training for 60 minutes per session, 3 times per week for 8 weeks. Physiological characteristics, allergic rhinitis symptoms, and cytokine secretions were comparatively analyzed before and after yoga training.

Results: After 8 weeks, the YOG group had increased peak nasal inspiratory flow (PNIF) and exhibited significantly decreased rhinitis symptoms and nasal blood flow (NBF) compared to pre-test. Moreover, the YOG group had significantly higher nasal secretion of interleukin (IL)-2 than the CON group.

Conclusion: The present findings demonstrated that 8 weeks of Hatha yoga training had beneficial effects in allergic rhinitis by improved clinical allergic rhinitis and cytokine profiles.

Key words: Allergic rhinitis, Yoga, Nasal blood flow, Peak nasal inspiratory flow, Cytokine

\section{Citation:}

Chanta, A., Klaewsongkram, J., Mickleborough, T. D., Tongtako, W. (2022). Effect of Hatha yoga training on rhinitis symptoms and cytokines in allergic rhinitis patients. Asian Pac J Allergy Immunol, 40(2), 126-133. https://doi.org/10.12932/ap-260419-0547

\footnotetext{
Affiliations:

${ }^{1}$ Faculty of Sports Science, Exercise Physiology in Special Population Research Group, Chulalongkorn University, Bangkok, Thailand

${ }^{2}$ Division of Allergy and Clinical Immunology,

Department of Medicine, Faculty of Medicine, Skin and

Allergy Research Unit, Chulalongkorn University, Bangkok, Thailand

Department of Kinesiology, School of Public Health,

Indiana University, Bloomington, Indiana, USA
}

\section{Corresponding author:}

Wannaporn Tongtako

Faculty of Sports Science, Chulalongkorn University

Rama 1 Rd, Patumwam, Bangkok 10330 Thailand

Email: Wannaporn_T@chula.ac.th

\section{Introduction}

Allergic rhinitis is defined as symptoms of sneezing, itching, nasal congestion, and rhinorrhea caused by immunoglobulin E (IgE)-mediated reactions against inhaled allergens and involving mucosal inflammation driven by type 2 helper T (Th2) cells, ${ }^{1}$ and constitutes a global health problem for all age groups. ${ }^{2}$ Allergens including pollen, house dust mites, and animal dander are the main causes of allergic reactions. ${ }^{3}$ The proliferation of allergen-stimulated $\mathrm{T}$ cells into Th2 cells can stimulate the release of cytokines, which can promote B-cell isotype switching. This subsequently induces local and systemic production of allergen-specific IgE by plasma cells, and results in mast cell proliferation and eosinophilic infiltration in the nasal mucosal epithelium. ${ }^{4}$ Inflammation associated with allergic rhinitis can reduce the physical size of the nasal passages by inducing vasodilatation, increasing blood flow, and increasing vascular permeability. The result is engorgement of nasal venous sinusoids, swelling of the anterior and inferior turbinates and obstruction of nasal airflow, ultimately contributing to nasal congestion. ${ }^{5}$ 
Various cytokines are secreted from various cells to control the inflammatory response, causing the accumulation of eosinophils in the nasal mucosa and the production of immunoglobulin. The interaction between immunoglobulin and allergens plays an important role in the inflammatory process, especially interleukin (IL)-6, a cytokine with an inflammatory effect in allergic rhinitis patients. ${ }^{6}$ In contrast, IL-2 is secreted by Th1 cells which is critical for supporting $\mathrm{T}$ cell activation, and the dominant physiological function of IL-2 signals in vivo is to restrain $\mathrm{T}$ cell activation and prevent autoimmunity. ${ }^{7}$ A previous study reported that in allergic rhinitis, an imbalance between Th1 and Th2 cells exists. ${ }^{8}$ The improvement in symptoms of allergen rhinitis was associated with differences in cytokine expression, such as IL-4 expression induced allergic rhinitis symptoms whereas IL-2 and IFN-gamma expression decreased the symptoms. ${ }^{9}$

The word "Yoga" is derived from Sanskrit, and means to "yoke or to join together" regarding which yoga has many types of practices. Hatha yoga is a type of yoga that is common practiced and is suitable for those who have never been trained to do it. Hatha yoga is the physical training part, combining postural exercise (asana), deep breathing (pranayama) and relaxation, and meditation (shavasana) and can profoundly influence health. ${ }^{10}$ There is mounting evidence that the mechanism of these beneficial effects on health is related in part to the impact of yoga on immune function ${ }^{11}$ and $c y-$ tokines. ${ }^{12}$ Regular practice of yoga promotes strength, endurance, and flexibility, and facilitates characteristics of friendliness, compassion, and greater self-control, while cultivating a sense of calmness and wellbeing. ${ }^{13}$ Yadav et al. demonstrated that after yoga training, there was a reduction in the mean levels of IL-6 in patients with chronic inflammatory diseases from the baseline to day 10. In addition, Vijayaraghava et al. demonstrated that yoga could reduce the extent of increase of TNF- $\alpha$ and IL-6. Moreover, Rajbhoj et al. showed that yoga practices could reduce pro-inflammatory cytokine levels and increase anti-inflammatory cytokine levels in industrial workers. ${ }^{14}$ Arai et al. ${ }^{15}$ reported that long-term exercise can enhance IL-2 production comparable to levels found in young male subjects indicating that chronic exercise could delay immunosenescence. Therefore, based on the available evidence, yoga training may be beneficial in terms of controlling rhinitis symptoms.

However, the effects of Hatha yoga training on rhinitis symptoms and cytokines in allergic rhinitis patients are still controversial. The purpose of the present study was to determine the effects of Hatha yoga training on rhinitis symptoms, nasal cytokine secretion, nasal blood flow (NBF), and peak nasal inspiratory flow (PNIF).

\section{Methods}

\section{Study design and procedure}

Sample size calculation was performed using $\mathrm{G}^{\star}$ Power (Univerity of Dusseldorf, Germany) at power $=0.8$ and effect size $=0.5$; a sample size of 20 patients would be required. Rhinitis patients were randomly assigned by the investigators into two groups: a sedentary control and Hatha yoga training group. During the study trial (8 weeks) the YOG group underwent a Hatha yoga training program, while the CON group continued with normal life and did not engage in exercise training. Pre- and post-study ( 8 weeks), body weight, body fat, body mass index, resting heart rate, and blood pressure were measured. In addition, pre- and post-study trial rhinitis symptoms, PNIF, nasal blood flow, and nasal secretions for cytokine analysis were evaluated. The research assistants and medical laboratory scientists who assessed the outcomes and performed blood biochemistry tests were blinded to the interventions.

\section{Participants}

Thirty allergic rhinitis patients aged 18-45 years, were recruited to this study from the Chulalongkorn University Health Service Center. All allergic rhinitis patients presented with clinical symptoms of persistent rhinitis (nasal congestion, sneeze, nasal itching, and running nose) for more than 4 days per week, and presented with a positive skin prick test (weal diameter $>3 \mathrm{~mm}$.) to house dust mites (Dermatophagoides pteronyssinus). Patients with asthma, chronic rhinosinusitis, hypertension, or cardiovascular diseases were excluded. Patients were asked to refrain from taking antihistamine medication for at least 3 days prior to testing, and to abstain from using oral and nasal steroids for at least 2 weeks prior to the start of the study. In addition, participants discontinued using leukotriene receptor antagonists for at least 1 week prior to testing. The participants were not to have undertaken a regular exercise program for at least 6 months prior to the start of the study.

All participants gave written informed consent prior to participation in the study. Medical and activity history was obtained via questionnaires. The study protocol was approved by the Institutional Review Board of the Health Sciences Group, Chulalongkorn University, (approval no. 102/2017). This study was registered as a clinical trial with clinical trials. gov (no. NCT03700944).

\section{Yoga training protocol}

The YOG group underwent a Hatha yoga training protocol for 60 minutes per session three times a week for 8 weeks at the Faculty of Sports Science, Chulalongkorn University, under the supervision of the primary investigators and a yoga instructor. The Hatha yoga training regimen consisted of 10 minutes of warm-up (I: seated mountain pose [dynamic], neck stretch pose, simple opener hip pose, and lateral twist pose; II: seated sun pose, boat pose [dynamic], spread leg forward bend, seated forward bend, and seat twist pose). This was followed by a workout for approximately $40 \mathrm{~min}$ including two sets, such as set I: mountain pose, standing sun pose, standing forward bend, lunge pose, push-up pose, lifted hip pose, upward facing dog pose, downward facing dog pose, lunge pose, standing forward bend, standing sun pose, and mountain pose (3 times/set) and set II: warrior pose, triangle pose, modified dancer pose, eagle pose, seated twist pose, head to knee pose, bow pose, cat pose, child pose, supine twist pose, half bridge pose, half shoulder stand pose, and fish pose (3 times/set), followed by a cool down for 10 minutes (meditation, alternate nostril breathing, and corpse pose). 


\section{General physiological characteristics}

The resting heart rate and blood pressure were measured using a heart rate monitor (Omron SEM-1 model, Japan). Body composition was measured using a bioelectrical impedance analyzer (InBody 220; Biospace, Seoul, Korea).

\section{Rhinitis symptom scores}

Nasal symptoms were recorded using the Total Nasal Symptom Score questionnaire. ${ }^{16}$ The patients were asked to score symptoms of persistent allergic rhinitis; nasal congestion, itching, sneezing, and rhinorrhea before and after 8 weeks. The total nasal symptom score was computed as the sum of four individual nasal symptom scores, which are the same as the four symptoms listed above. The scores ranged from 0 to $3(0=$ none, $1=$ mild, $2=$ moderate, $3=$ severe $)$.

\section{Peak nasal inspiratory flow}

PNIF was measured using a PNIF meter (In-Check Oral; Clement Clark International, UK.) attached to an anesthesia mask. During the procedure, the participants wore a mask over the nose and mouth, which was connected to a plastic cylinder through which the air passed during inspiration; participants inspired forcefully through the nose, with lips tightly closed. Inside the cylinder, there is a diaphragm that moves according to the airflow, and the maximum peak flow is registered in a scale ranging from $30-370 \mathrm{~L} / \mathrm{min}$. PNIF was measured before and after exercise.

\section{Nasal blood flow}

NBF was measured by laser Doppler flowmetry (DRT4; Moor instruments, UK.). All participants were advised to breathe normally and not to cough, speak, or move during the test. A side delivery endoscopic probe with a flexible nylon sleeve with a diameter of $1.34 \mathrm{~mm}$ was placed on the anterior surface of the nose.

\section{Nasal secretion collection and handing}

Nasal secretions collection was performed bilaterally with filter paper strips $(7 \times 30 \mathrm{~mm}$ Whatman No. 42 ; Whatman, Clifton, NJ). Three filter paper strips were sequentially placed on each anterior portion of the inferior turbinate for $10 \mathrm{~min}$ utes. These filter paper strips were collected into appropriate tubes and centrifuged at 3,000 rpm for 5 minutes at $4^{\circ} \mathrm{C}$ and immediately frozen at $-70^{\circ} \mathrm{C}$ until later analysis

\section{Cytokine analysis}

Nasal secretion levels of the cytokines IL-2 and IL-6 were determined using the sandwich ELISA technique. ${ }^{17}$ The data were collected by a medical technologist. Quantitative anti-human IL-2 ELISA was conducted using the Human IL-2 High Sensitivity ELISA kit (Invitrogen, Catalog Number BMS221HS) and anti-human IL-6 ELISA was conducted using the Human IL-6 High Sensitivity ELISA kit (Invitrogen, Catalog Number BMS213HS). In brief, the percolated of anti-human IL- 2 or IL-6 plates were washed twice with wash buffer. The human IL-2 standard dilutions ranging from 0.94 to $60 \mathrm{pg} / \mathrm{ml}$ or IL-6 standard dilutions ranging from 0.08 to $5 \mathrm{pg} / \mathrm{ml}$ were loaded $100 \mu \mathrm{L}$ in each standard well. Then, the sample diluent $(50 \mu \mathrm{L})$ was added and $50 \mu \mathrm{L}$ of each nasal fluid sample was loaded into the sample wells. To the blank wells were added $100 \mu \mathrm{L}$ of sample diluent. Next, $50 \mu \mathrm{L}$ of the Biotin-Conjugate anti-human IL- 2 or IL- 6 was added to all wells. After 2 hours incubation at RT and washing, $100 \mu \mathrm{L}$ of streptavidin-HRP was added to all wells and incubated at RT for 1 hour. Plates were washed, and $100 \mu \mathrm{L}$ of Amplification Solution was added to all wells and incubated at RT for exactly 15 minutes. Then, the plates were washed, and $100 \mu \mathrm{L}$ of the Amplification Solution II was added and incubated at RT for exactly 30 minutes. To develop color, $100 \mu \mathrm{L}$ of the TMB substrate solution was added for 10$20 \mathrm{~min}$. The plates were read by the ELISA reader at $620 \mathrm{~nm}$. The sensitivity was determined to be $0.40 \mathrm{pg} / \mathrm{ml}$ for anti-human IL-2 and $0.03 \mathrm{pg} / \mathrm{ml}$ for anti-human IL-6.

\section{Statistical analysis}

Statistical analysis was performed using SPSS version 22 for Windows (SPSS Inc., Chicago, USA). The normality of the distribution of the variables was tested using the Shapiro-Wilk test. The dependent variables between pre-test and post-test were analyzed using a paired t-test. The dependent variables between the CON and YOG groups were analyzed using an independent t-test. Differences were considered significant at $p<0.05$. Data were expressed as mean \pm standard deviation (SD).

\section{Results}

As shown in Figure 1, the eligible participants were randomly allocated into two groups: CON $(n=14)$ and YOG $(n=13)$. A total of 3 participants withdrew from the study. One and two participants withdrew from the CON and YOG groups due to scheduling difficulties. Therefore, the CON and YOG groups comprised 14 (3 male, 11 female) and 13 (3 male, 10 female) participants, respectively.

\section{General physiological characteristics}

General characteristics of the participants are shown in Table 1. There were no significant differences in body weight, body mass index, body fat, resting heart rate, and blood pressure between pre and post- test and between the CON and YOG groups.

\section{Rhinitis symptoms}

After 8 weeks, the YOG group had experienced significant decreases in rhinitis symptoms, such as nasal congestion (2.38, 1.07, $p=0.001 ; 2.14,1.07, p=0.001)$, itching (2.23, $1.23, p=0.000 ; 2.07,1.23, p=0.000)$, sneezing $(2.23,1.55, p=$ $0.003 ; 2.14,1.55, p=0.000)$, rhinorrhea $(2.46,1.23, p=0.000$; $2.14,1.23, p=0.002)$ and total rhinitis symptoms $(9.31,4.69$, $p=0.000 ; 8.57,4.69, p=0.000)$ when compared with the pretest and CON groups (Table 2).

\section{Peak nasal inspiratory flow and nasal blood flow}

After 8 weeks, the YOG groups showed significantly increased $(60.00,106.15, p=0.001 ; 66.43,106.15, p=0.03)$ PNIF (Figure 2A) and significantly decreased (284.67, 191.39, $p=0.000 ; 266.53,191.39, p=0.001$ ) NBF (Figure 2B) compared with the pre-test and CON groups. 


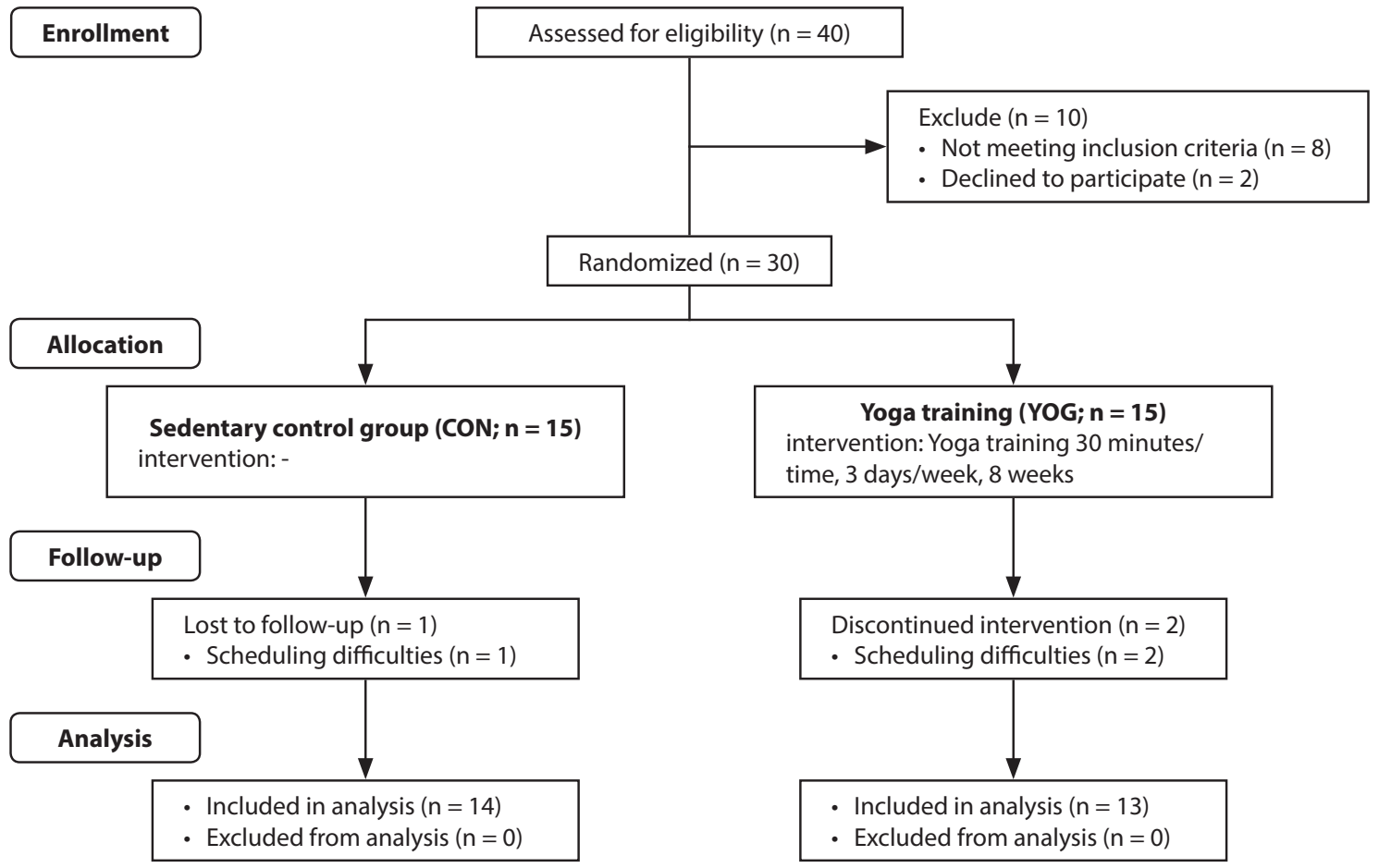

Figure 1. CONSORT 2010 flow diagram of participant allocation, follow-up and analysis.

Table 1. The comparison of the general physiological characteristics variables between control group (CON) and yoga training group (YOG).

\begin{tabular}{|lcccc|}
\multirow{2}{*}{\multicolumn{1}{c}{ Variables }} & \multicolumn{2}{c}{ CON $(\mathbf{n}=\mathbf{1 4})$} & \multicolumn{2}{c|}{ YOG $(\mathbf{n}=13)$} \\
\cline { 2 - 5 } & Pre & Post & Pre & Post \\
\hline Body weight $(\mathrm{kg})$ & $58.62 \pm 10.39$ & $58.86 \pm 10.67$ & $63.15 \pm 19.46$ & $62.20 \pm 19.14$ \\
\hline BMI $\left(\mathrm{kg} / \mathrm{m}^{2}\right)$ & $21.67 \pm 2.99$ & $21.74 \pm 3.14$ & $23.23 \pm 4.90$ & $23.22 \pm 4.95$ \\
\hline Body Fat $(\%)$ & $25.60 \pm 4.71$ & $24.88 \pm 5.23$ & $26.43 \pm 5.92$ & $25.47 \pm 6.68$ \\
\hline Resting HR (b/min.) & $75.14 \pm 9.68$ & $75.64 \pm 10.85$ & $74.46 \pm 6.55$ & $75.85 \pm 10.33$ \\
\hline Systolic BP $(\mathrm{mmHg})$ & $114.57 \pm 18.18$ & $120.29 \pm 21.45$ & $111.62 \pm 10.51$ & $113.38 \pm 13.14$ \\
\hline Diastolic BP $(\mathrm{mmHg})$ & $74.07 \pm 7.88$ & $76.86 \pm 10.48$ & $76.69 \pm 5.81$ & $75 \pm 8.71$ \\
\hline
\end{tabular}

Data are presented as mean $\pm \mathrm{SD}$. $\mathrm{BMI}=$ Body Mass Index, $\mathrm{HR}=$ Heart rate, $\mathrm{BD}=$ Blood pressure

Table 2. The comparison of the rhinitis symptoms variables between control group (CON) and yoga training group (YOG).

\begin{tabular}{lcccc}
\multirow{2}{*}{ Variables } & \multicolumn{2}{c}{ CON $(\mathbf{n}=\mathbf{1 4})$} & \multicolumn{2}{c}{ YOG $(\mathbf{n}=13)$} \\
\cline { 2 - 5 } & \multicolumn{1}{c}{ Pre } & Post & Pre & Post \\
\hline Nasal congestion & $2.21 \pm 0.43$ & $2.14 \pm 0.66$ & $2.38 \pm 0.50$ & $1.07 \pm 0.86^{* \dagger}$ \\
\hline Itching & $2.00 \pm 0.39$ & $2.07 \pm 0.53$ & $2.23 \pm 0.44$ & $1.23 \pm 0.44^{* \dagger}$ \\
Sneezing & $2.07 \pm 0.47$ & $2.14 \pm 0.53$ & $2.23 \pm 0.60$ & $1.55 \pm 0.69^{* \dagger}$ \\
Rhinorrhea & $2.36 \pm 0.50$ & $2.14 \pm 0.66$ & $2.46 \pm 0.51$ & $1.23 \pm 0.73^{* \dagger}$ \\
\hline Total of symptoms & $8.64 \pm 1.00$ & $8.57 \pm 2.10$ & $9.31 \pm 1.25$ & $4.69 \pm 1.43^{* \dagger}$ \\
\hline
\end{tabular}

Data are presented as mean $\pm \mathrm{SD} .{ }^{*} p<.05$, vs. pre-test ${ }^{\dagger} p<.05$, vs. CON 

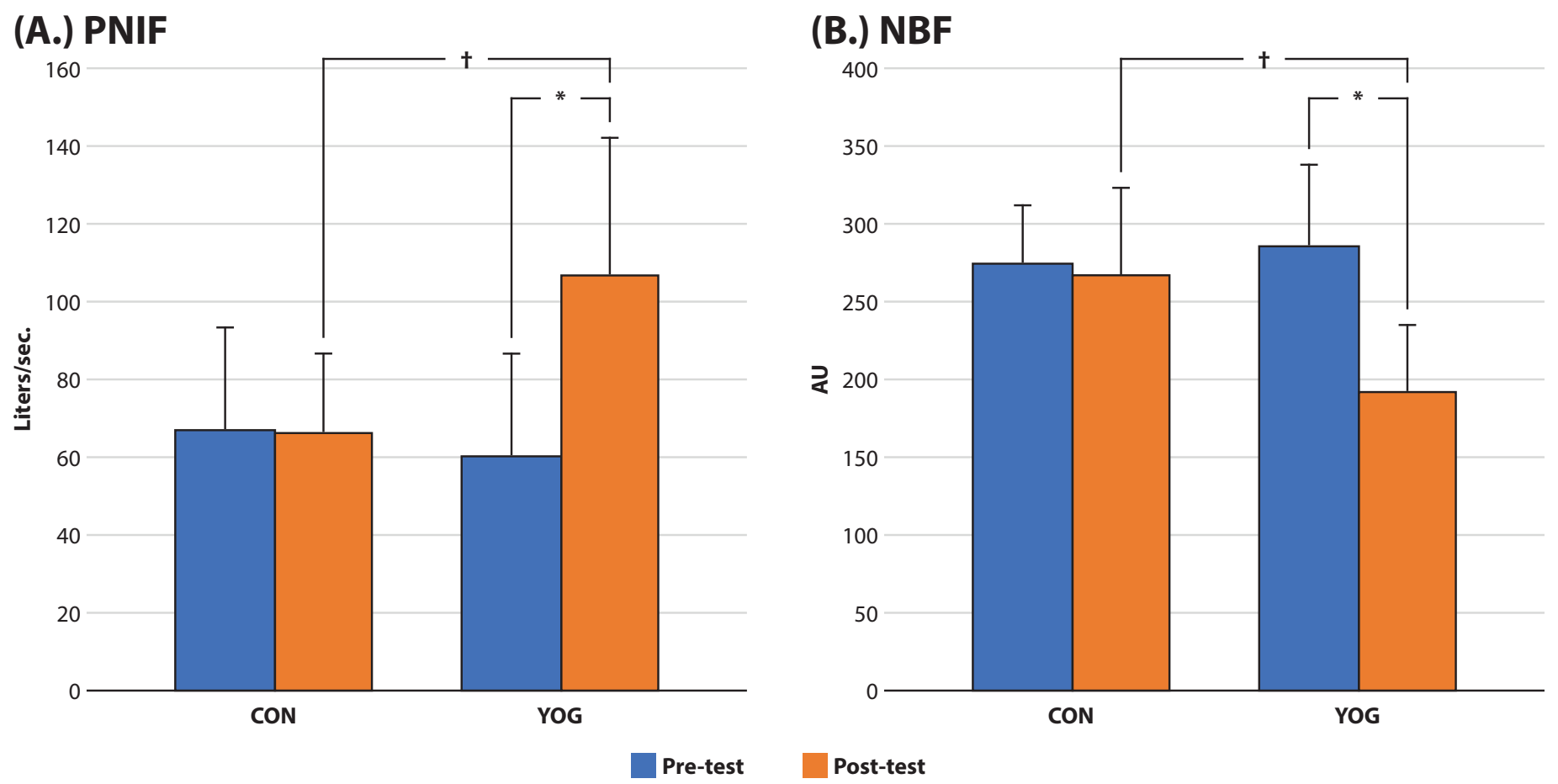

Figure 2. The comparison of peak nasal inspiratory flow (PNIF) (A.) and nasal blood flow (NBF) (B.) between pre- and post-training in the CON and the YOG group and between groups.

Data are presented as mean \pm SD. ${ }^{\star} p<.05$, vs. pre-test ${ }^{\dagger} p<.05$, vs. CON

(A.) IL-2

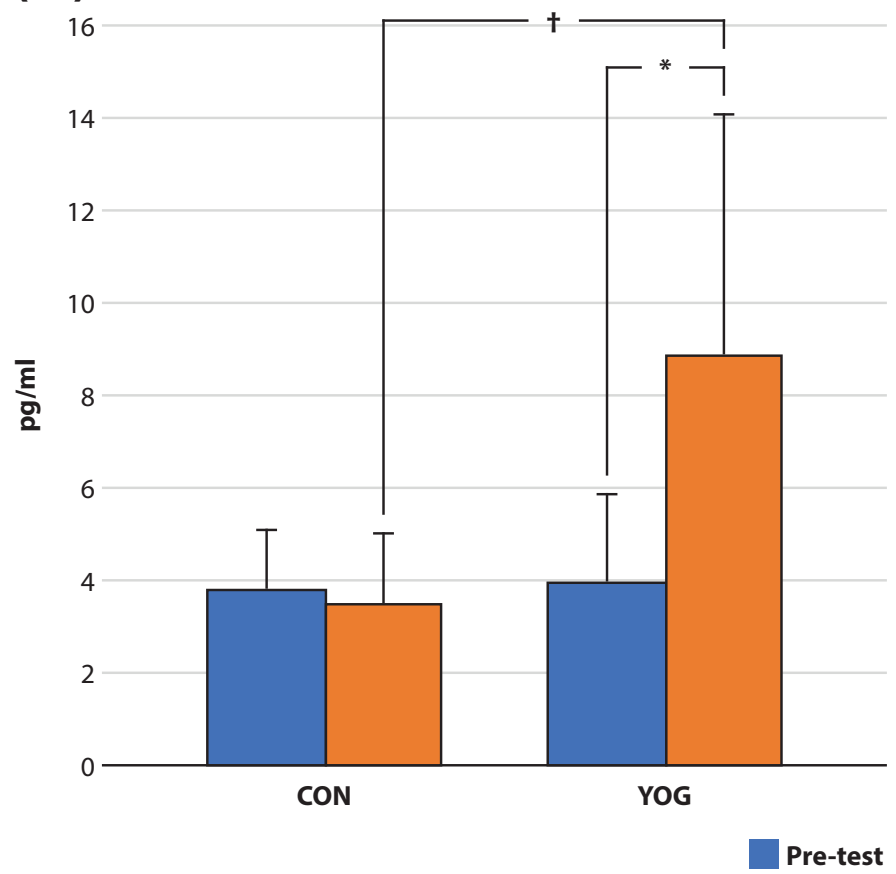

(B.) IL-6

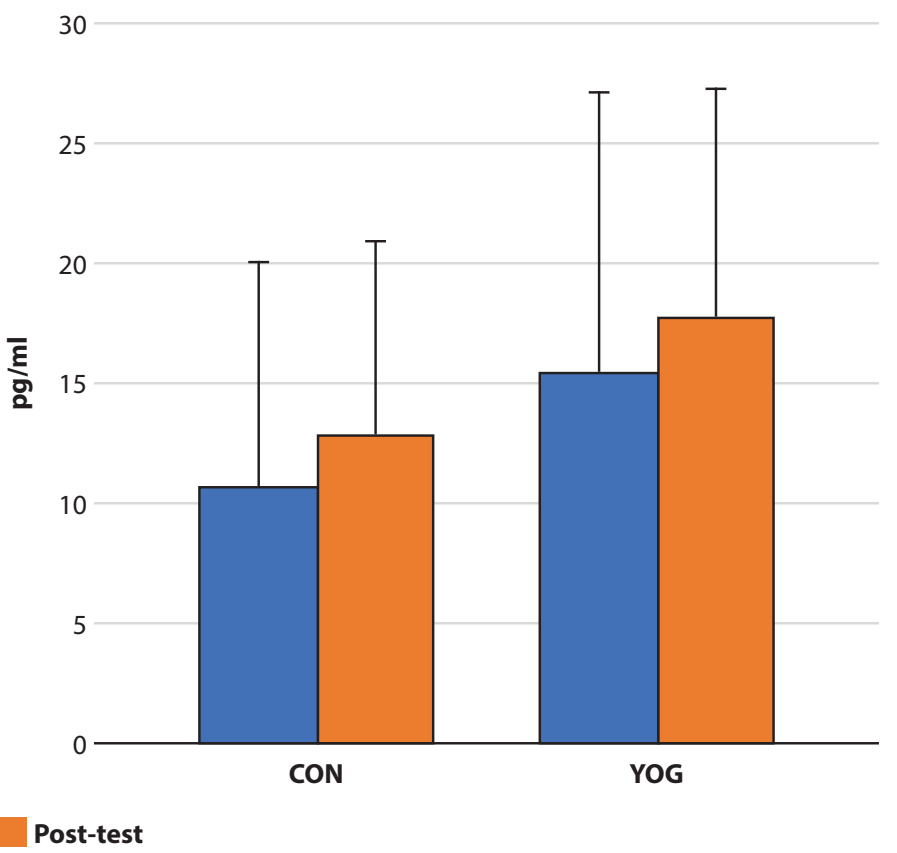

Figure 3. The comparison of cytokine levels in nasal secretion between pre- and post-training in the CON and the YOG group and between groups.

Data are presented as mean $\pm \mathrm{SD} .{ }^{\star} p<.05$, vs. pre-test ${ }^{\dagger} p<.05$, vs. CON 


\section{Cytokine determination}

At pre-test, there were no significant differences in IL-2 (Figure 3A) and IL-6 (Figure 3B) levels between the two groups. After 8 weeks, the YOG group had significantly higher baseline IL-2 levels $(3.419,8.87, p=0.019)$ than the CON group. Furthermore, the YOG group had significantly increased IL-2 levels $(3.94,8.87, p=0.024)$ compared with pretest as shown in Figure $\mathbf{3 A}$.

\section{Discussion}

\section{Physiological variables}

The results showed that after 8 weeks of Hatha yoga training, there was no difference in physiological variables including body weight, body mass index, resting heart rate, and systolic and diastolic blood pressure between the CON and YOG groups, and also between before and after the experiment. The results were supported by existing reports such as Santalla et al. ${ }^{18}$ who studied the effect of yoga training twice a week for 4 months in elderly people and showed that physiological variables including body weight, body mass index, resting heart rate, and blood pressure were not changed. In addition, Yonglitthipagon et al. ${ }^{19}$ demonstrated that yoga training for 30 minutes twice a week for 12 weeks resulted in reduced menstrual pain and improved quality of life among women with menstrual pain. However, there was no effect on body weight and body mass. Moreover, Cheema et al. ${ }^{20}$ found that body weight, body mass index, resting heart rate, systolic and diastolic blood pressure of office staff who received yoga training for 50 minutes 3 times a week for 10 weeks were not different from those in the control group. An additional explanation could be that the yoga training program of the present study consisted of Pranayama and meditation Asana using the Hatha Yoga method, which emphasizes breathing and posture to balance the body for relaxation and concentration. The lack of change in physiological variables may be due to the fact that yoga is not a high-intensity exercise that can increase the body's metabolism and improve the tolerance of the circulatory system, thereby directly affecting physiological parameters. Instead, yoga is a relaxation exercise for increasing flexibility and involves breathing exercises.

\section{Rhinitis symptoms}

In our study, the rhinitis symptom scores were significantly different in both Hatha yoga training and usual life groups. The volume of nasal inspiratory flow increased. PNIF testing is a reliable and acceptable method of assessing respiratory obstruction and nasal cavity inflammation, which can be used for healthy and allergic rhinitis patients. ${ }^{21}$ Congruent to the empirical evidence, it was found that the nasal cavity resistance of allergic rhinitis patients who received Hatha yoga training for 3 months was decreased, ${ }^{22}$ and PNIF was increased after exercise. ${ }^{23}$ The mechanism by which training exercise improves nasal rhinitis symptoms can be explored as a sympathetic nervous system activated nasal vasoconstriction that reduces the volume of the venous sinusoids, which causes the maximum airflow volume in the nasal cavity to increase. ${ }^{24}$ Yoga is a mind-body exercise in which both physical and mental disciplines are brought together to achieve peacefulness of mind and body, resulting in a relaxed state, i.e., relaxation of breathing muscles. In addition, yoga stimulates pulmonary stretch receptors by expanding the lungs through the relaxation of the smooth muscles of the larynx and the bronchial branches resulting in the expansion of air channels and reduction of air resistance in the air ducts and nasal cavity. ${ }^{25}$ Therefore, allergic rhinitis patients who underwent Hatha yoga training had clear nostrils.

In contrast, nasal blood flow, nasal congestion, itching, sneezing, rhinorrhea, and total symptoms at baseline were decreased $(p<.05)$. The mechanism of nasal symptom generation in allergic rhinitis is more complex. Nasal congestion in allergic rhinitis is caused by swelling of nasal cavity tissue, narrowing the nasal cavity and increasing secretion as a result of the interaction between allergens and immunoglobulins. ${ }^{26}$ Yoga reduces the volume of blood flowing through the nasal vascular mucosa, which reduces swelling and nasal cavity resistance. Studies relevant to yoga and nasal blood flow are not completely expressed. It has been thought that nasal blood flow increases when the nasal septum is inflamed, causing the rich blood supply of the nose. Nasal passages become red and swollen, which is the main symptom of rhinitis patients. ${ }^{27}$ A study demonstrated that isotonic exercise effectively reduces the resistance of the nasal cavity and decreases nasal cavity blood flow. ${ }^{28}$ Similar to the results reported by Nair, ${ }^{29}$ nasal breathing exercise causes the air to sway, which in turn increases the exchange of air between the sinuses and nasal cavity with decreased nasal nitric oxide, which could have resulted in the improved rhinitis symptoms. The improvement is most likely related to Pranayama, which is an integral part of Hatha yoga. As a deep breathing technique that reduces dead space ventilation and work of breathing, regular practice of Pranayama improves the respiratory functions by refreshing the air throughout the lungs and clearing of the nostril. ${ }^{30}$ Another mechanism could be as shown in our previous study, which demonstrated that 8 weeks of moderate aerobic exercise training led to reduced allergic rhinitis symptoms by decreased nasal resistance due to the reduced blood flow, leading to reduced nasal congestion. ${ }^{31}$ This relates to Hatha yoga training which have intensity in the period of low to moderate intensity.

In the present study, it was clearly seen that nasal symptoms, PNIF, and nasal blood flow in patient who underwent Hatha yoga training were significantly improved compared to baseline symptoms and significantly better than data among the control patients.

\section{Cytokine determination}

IL-2 levels in the nasal secretion of the yoga group were significantly higher after training than before training and were also higher than in the control participants, but IL-6 showed no difference in both groups.

Cytokines are small glycoproteins secreted by white blood cells and various cell types in response to a number of stimuli. Cytokines act on target cells by capturing the receptors on the cell surface and stimulate the change within the cells. ${ }^{32} \mathrm{~A}$ previous study confirmed that cytokines are important factors in the pathology and mechanism of allergic rhinitis. ${ }^{33}$ IL-2 is a cytokine signaling molecule produced by T helper-1 lymphocytes in the immune system, which is composite with 
$\mathrm{T}$ helper-2 lymphocytes and important in the intracellular destruction of phagocytosed microbes. ${ }^{34}$ A previous study found that IL-2, IL-4, IL-13, and TNF- $a$ levels in nasal secretions of allergic rhinitis patients who performed exhaustive and moderate intensity exercise were significantly higher than those among healthy people. ${ }^{31}$ In the present study, IL-2 levels in nasal secretion increased in patients following yoga training compared to those in the control group. Arai et al. ${ }^{15}$ demonstrated that long-term exercise training could enhance IL-2 production, indicating that chronic exercise could delay immunosenescence. Based on our data, we suggest that the improvement in clinical symptoms in allergic rhinitis patients may be a consequence of cytokine deviation after yoga training. However, in the present study, there were no significant differences in IL-6 levels after 8 weeks of yoga training when compared to pre-test and the CON group. IL- 6 acts as both a pro-inflammatory cytokine and an anti-inflammatory myokine; its production is associated with an antigen-induced late nasal response. Several studies have illustrated that yoga reduces IL-6 levels, ${ }^{34}$ which in contrast to our study may be due to the short duration of yoga training (just 8 weeks). Although the levels of IL-6 did not change in this study, the IL-2 level increased, indicating a reduction in inflammation from allergic rhinitis. The reduced inflammation resulting from yoga may be because yoga increases the level of antioxidants, which causes the reduction of free radicals that contain oxygen to reactive oxygen species; ${ }^{35}$ this is relevant to the mechanism of inflammation prevention and cytokine levels.

The present study demonstrates that Hatha yoga training for 60 minutes per session three times a week for 8 weeks has beneficial effects in allergic rhinitis patients by reducing rhinitis symptoms. The extensive benefits on immune function were to improve cytokine deviation by increasing IL-2 levels.

\section{Acknowledgements}

We are indebted to all volunteers. We would like to thank Supranee Buranapraditkun, Ph.D. for cytokines analysis. This study was supported by The $90^{\text {th }}$ Anniversary Chulalongkorn University Fund (Ratchadaphiseksomphot Endowment Fund), Chulalongkorn University and Faculty of Sports Science Fund, Chulalongkorn University.

\section{Conflicts of interest}

The authors declare that they have no competing interests.

\section{Author contribution}

- A.C. designed and performed the experiments, data collection, and analyzed the data and drafted the manuscript.

- J.K. designed the experiments, contributed to sample preparation, aided in interpreting the results.

- T.D.M designed the experiments and reviewed the manuscript.

- W.T. conceptualized and designed the study, assisted with the implemented of the intervention, data collection, and data analysis, interpreting the results, discussion and conclusion, and revised/reviewed the manuscript.

- All authors have read and approved the final version of the manuscript, and agree with the order of presentation of the authors.

\section{References}

1. Bousquet J, Khaltaev N, Cruz AA, Denburg J, Fokkens WJ, Togias A, et al. Allergic rhinitis and its impact on asthma (ARIA) 2008. Allergy. 2008;86:8-160.

2. Bousquet J, Schunemann HJ, Samolinski B, Demoly P, Baena-Cagnani CE, Bachert C, et al. Allergic Rhinitis and its Impact on Asthma (ARIA): achievements in 10 years and future needs. J Allergy Clin Immunol. 2012;130:1049-62.

3. Mygind N. Allergic rhinitis. Chem Immunol Allergy. 2014;100:62-8.

4. Dykewicz MS, Hamilos DL. Rhinitis and sinusitis. J Allergy Clin Immunol. 2010;125:S103-15.

5. Gosset P, Malaquin F, Delneste Y, Wallaert B, Capron A, Joseph M, et al. Interleukin-6 and interleukin-1 alpha production is associated with antigen-induced late nasal response. J Allergy Clin Immunol. 1993;92: 878-90.

6. Zhao N, Liu HJ, Sun YY, Li YZ. Role of interleukin-6 polymorphisms in the development of allergic rhinitis. Genet Mol Res. 2016;15:gmr6987.

7. Ma A, Koka R, Burkett P. Diverse functions of IL-2, IL-15, and IL-7 in lymphoid homeostasis. Annu Rev Immunol. 2006;24:657-79.

8. König K, Klemens C, Eder K, San Nicoló M, Becker S, Kramer MF, et al. Cytokine profiles in nasal fluid of patients with seasonal or persistent allergic rhinitis. Allergy Asthma Clin Immunol. 2015;11:26.

9. Fireman P. Cytokines and allergic rhinitis. Allergy Asthma Proc. 1996;17:175-8.

10. Villien F, Yu M, Barthélémy P, Jammes Y. Training to yoga respiration selectively increases respiratory sensation in healthy man. Respir Physiol Neurobiol. 2005;146:85-96.

11. Field T. Yoga clinical research review. Complement Ther Clin Pract. 2011;17:1-8.

12. Vijayaraghava A, Doreswamy V, Narasipur OS, Kunnavil R, Srinivasamurthy N. Effect of yoga practice on levels of inflammatory markers after moderate and strenuous Exercise. J Clin Diagn Res. 2015; 9:Cc08-12.

13. Shruti A, Surya K, Kumar VV, Kumar MS, Sarika P. Role of jalaneti and pranayama in allergic rhinitis with asthma. Int J Yoga - Philosop Psychol Parapsychol. 2016;4:3-7.

14. Rajbhoj PH, Shete SU, Verma A, Bhogal RS. Effect of yoga module on pro-inflammatory and anti-inflammatory cytokines in industrial workers of lonavla: a randomized controlled trial. J Clin Diagn Res. 2015;9: Cc01-5.

15. Arai MH, Duarte AJ, Natale VM. The effects of long-term endurance training on the immune and endocrine systems of elderly men: the role of cytokines and anabolic hormones. Immun Ageing. 2006;3:9.

16. Chusakul S, Phannaso C, Sangsarsri S, Aeumjaturapat S, Snidvongs K. House-dust mite nasal provocation: a diagnostic tool in perennial rhinitis. Am J Rhinol Allergy. 2010;24:133-6.

17. Aydin S. A short history, principles, and types of ELISA, and our laboratory experience with peptide/protein analyses using ELISA. Peptides. 2015;72:4-15.

18. Santaella DF, Devesa CRS, Rojo MR, Amato MBP, Drager LF, Casali KR, et al. Yoga respiratory training improves respiratory function and cardiac sympathovagal balance in elderly subjects: a randomised controlled trial. BMJ Open. 2011;24:e000085.

19. Yonglitthipagon P, Muansiangsai S, Wongkhumngern W, Donpunha W, Chanavirut R, Siritaratiwat W, et al. Effect of yoga on the menstrual pain, physical fitness, and quality of life of young women with primary dysmenorrhea. J Bodyw Mov Ther. 2017;21:840-6.

20. Cheema BS, Houridis A, Busch L, Raschke-Cheema V, Melville GW, Marshall PW, et al. Effect of an office worksite-based yoga program on heart rate variability: outcomes of a randomized controlled trial. BMC Complement Altern Med. 2013;13:82.

21. Teixeira RU, Zappelini CE, Alves FS, da Costa EA. Peak nasal inspiratory flow evaluation as an objective method of measuring nasal airflow. Braz J Otorhinolaryngol. 2011;77:473-80.

22. Chellaa R, Soumya MS, Inbaraj G, Nayar R, Saidha PK, Menezes VH, et al. Impact of hatha yoga on the airway resistances in healthy individuals and allergic rhinitis patients. Indian J Otolaryngol Head Neck Surg. 2017; doi:10.1007/s12070-017-1098-1

23. Tongtako W, Klaewsongkram J, Jaronsukwimal N, Buranapraditkun S, Mickleborough TD, Suksom D. The effect of acute exhaustive and moderate intensity exercises on nasal cytokine secretion and clinical symptoms in allergic rhinitis patients. Asian Pac J Allergy Immunol. 2012;30:185-92. 
24. Olson LG, Strohl KP. The response of the nasal airway to exercise. Am Rev Respir Dis. 1987;135:356-9.

25. Mandanmohan L, Jatiya L, Udupa K, Bhavanani AB. Effect of yoga training on handgrip, respiratory pressures and pulmonary function. Indian J Physiol Pharmacol. 2003;47:387-92.

26. Okubo K, Kurono Y, Ichimura K, Enomoto T, Okamoto Y, Kawauchi H, et al. Japanese guidelines for allergic rhinitis 2017. Allergol Int. 2017;66: 205-19.

27. Busse WW, Holgate ST. Asthma and Rhintis. Combridge, MA: Blackwell Scientific Publications, 1995.

28. Clarke RW. The differential effect of isotonic and isometric exercise on nasal blood flow as measured by laser doppler analysis. Otolaryngol Head Neck Surg. 1996;115:130.

29. Nair S. Nasal breathing exercise and its effect on symptoms of allergic rhinitis. Indian J Otolaryngol Head Neck Surg. 2012;64:172-76.
30. Soumya M, Chellaa R,Inbaraj. Hatha yoga effect on airway resistances of tobacco smokers with allergic rhinitis. J Yoga \& Physio. 2017;2:555594.

31. Tongtako W, Klaewsongkram J, Mickleborough TD, Suksom D. Effects of aerobic exercise and vitamin C supplementation on rhinitis symptoms in allergic rhinitis patients. Asian Pac J Allergy Immunol. 2018;36:222-31.

32. Scadding G. Cytokine profiles in allergic rhinitis. Curr Allergy Asthma Rep. 2014;14:435.

33. Ngoc LP, Gold DR, Tzianabos AO, Weiss ST, Celedón JC. Cytokines, allergy, and asthma. Curr Opin Allergy Clin. 2005;5:161-6.

34. Netam R, Yadav RK, Khadgawat R, Sarvottam K, Yadav R. Interleukin-6, vitamin $\mathrm{D} \&$ diabetes risk-factors modified by a short-term yoga-based lifestyle intervention in overweight/obese individuals. Indian J Med Res. 2015;141:775-82.

35. Patil SG, Dhanakshirur GB, Aithala MR, Naregal G, Das KK. Effect of yoga on oxidative stress in elderly with grade-I hypertension: a randomized controlled study. J Clin Diagn Res. 2014;87:Bc04-7. 